Cahiers de recherches médiévales

\title{
Servir? La noblesse française face aux sollicitations militaires du roi
}

(fin du règne de Saint Louis-fin du règne de Philippe le Bel)

\section{Xavier Hélary}

\section{(2) OpenEdition}

\section{Journals}

Édition électronique

URL : https://journals.openedition.org/crm/745

DOI : $10.4000 / \mathrm{crm} .745$

ISSN : 1955-2424

\section{Éditeur}

Honoré Champion

Édition imprimée

Date de publication : 30 décembre 2006

Pagination : 21-40

ISSN : 1272-9752

Référence électronique

Xavier Hélary, «Servir? La noblesse française face aux sollicitations militaires du roi », Cahiers de recherches médiévales [En ligne], 13 | 2006, mis en ligne le 27 novembre 2009, consulté le 15 décembre 2022. URL : http://journals.openedition.org/crm/745 ; DOI : https://doi.org/10.4000/crm.745 


\section{rM}

\section{Servir? \\ La noblesse française face aux sollicitations militaires du roi (fin du règne de Saint Louis - fin du règne de Philippe le Bel)}

On examine habituellement les questions liées au service militaire rempli par la noblesse du point de vue du roi. Les sources, de fait, y inclinent et, fort légitimement, c'est le renforcement tendanciel du pouvoir royal (dont l'aspect militaire est peut-être la facette la plus importante, d'autant que la fiscalité lui est étroitement liée) qui a prioritairement intéressé les historiens. Cependant, à n'adopter que ce point de vue, on risque de n'apercevoir qu'une partie de la réalité, et d'abord parce que les documents produits par le gouvernement royal ont souvent une valeur avant tout normative, particulièrement sans doute dans ces deux domaines si proches que sont la fiscalité et la levée des armées. L'objet de cet article est de rassembler quelques éléments d'explication sur la réception, par la noblesse française, à laquelle elles s'adressaient prioritairement, des sollicitations royales en matière militaire, entre la croisade de Tunis et la fin du règne de Philippe le Bel, une période reconnue depuis longtemps comme décisive dans la mutation du recrutement des armées ${ }^{1}$. Il s'agit du coup d'examiner les motivations qui ont pu pousser les combattants à servir dans les armées royales, et à évaluer la pertinence du stéréotype volontiers mis en avant par l'éthique nobiliaire, le service du roi, et la place relative qu'il a pu jouer dans la constitution des armées royales à cette époque ${ }^{2}$.

Un premier point doit être précisé d'emblée. Dans la période envisagée, aucune roi de France n'a eu à déplorer la faiblesse de ses effectifs. Un chroniqueur du temps avance que Philippe III, lors de l'expédition de Foix, «mena grant foison de bonne chevalerie aveques lui ». «Touz les chans estoient couvers de genz », affirmet-il, à propos de l'ost de Sauveterre en $1276^{3}$. Cette constatation vaut pour toutes les autres expéditions menées par l'armée royale à cette époque. De l'avis général, les deux croisades de Saint Louis n'échouèrent nullement en raison d'un manque de

${ }^{1}$ C'était l'objet de ma thèse de doctorat, intitulée L'ost de France. La guerre, les armées, la société politique au royaume de France (fin du règne de Saint Louis - fin du règne de Philippe le Bel), soutenue en décembre 2004 à l'université Paris-Sorbonne (Paris IV). C'est pour moi l'occasion de remercier mes maîtres Philippe Contamine et Jacques Verger.

${ }^{2}$ Philippe Contamine, «Noblesse et service : l'idée et la réalité dans la France de la fin du Moyen Âge», Nobilitas. Funktion und Repräsentation des Adels in Alteuropa, s. d. Otto Gerhard Oexle et Werner Paravicini, Göttingen, 1997 (Veröffentlichungen des Max-PlanckInstituts für Geschichte, 133), p. 298-311; et, sur un plan plus global, l'article classique du même, "Points de vue sur la chevalerie en France à la fin du Moyen Âge », Francia, 4 (1976), p. 255-285. Voir également les aperçus donnés sur le long terme par André Corvisier, "Quelques réflexions sur devoir militaire et service militaire », Des étoiles et des croix. Mélanges offerts à Guy Pedroncini, préparés s. d. Jean-Claude Allain, Economica, Paris, 1995, p. 31-39. La question des motivations des chevaliers n'a guère intéressé les historiens, sinon pour le cas bien particulier de la croisade, à l'exemple de Jean Flori, Pierre l'Ermite et la première croisade, Paris, Fayard, 1999, aux chapitres X et XI, p. 179-225.

${ }^{3}$ Chronique anonyme finissant en 1286, Recueil des historiens de Gaule et de la France (désormais RHGF), t. XXI, p. 92 et 93.

Cahiers de Recherches Médiévales, 13, 2006 
combattants. Philippe III, ni à Sauveterre (1276) ni en Catalogne (1285), ne se trouva confronté à un ennemi supérieur en nombre. Quant à Philippe le Bel, une fois épuisés ses faciles succès de la décennie 1290, c'est après la sanglante défaite de Courtrai (1302) qu'il rassembla l'armée la plus nombreuse de son règne. Dans les années terribles 1302-1304, l'armée royale put toujours aligner des effectifs équivalents à ceux des Métiers flamands, qui combattaient eux au plus près de leur base. Jamais les chroniqueurs ni même les documents produits par la propagande royale, qui ne manquait pourtant pas d'imagination, n'invoquèrent un surclassement numérique pour expliquer l'insuccès, au cours de ces deux années, des armées de Philippe le $\mathrm{Bel}^{4}$.

Une seule anecdote vient contredire en apparence cette constatation. C'est le dépit que le chroniqueur Primat prête à Saint Louis arrivant, au printemps 1270, à Aigues-Mortes, d'où il devait partir pour Tunis :

Et le courage du roy très crestien estoit si diligent et si très ardant el negoce de la croyz, que un jour que il chevauchoit avant que il venist au port, il regarda entour lui pour veoir sa gent, qui avec li estoient ; et donc commença à souspirer, quer il li estoit avis que il estoient trop pou ; et puis se retourna devers l'evesque de Paris, qui chevauchoit le plus prochain de lui, et li dist: «Las! Comme je voi pou de gens au jour d'ui qui aient a cuer le besoing de la croiz ne de la sainte Église $»^{5}$.

L'anecdote sonne juste, et elle correspond bien au portrait qu'ont dressé de Saint Louis les différents chroniqueurs. Mais c'est bien sur le compte de l'exigence extrême de Saint Louis qu'il faut mettre ce dépit que, Primat le précise plus loin, la réalité ne justifiait pas. On sait, de fait, que les effectifs de l'armée croisée furent jugés tout à fait suffisants - sans doute autour de vingt mille hommes, à qui il fallut faire traverser la Méditerranée.

Une seconde remarque liminaire doit être faite. L'armée royale ne connut jamais de révolte. La seule qu'on connaisse en un demi-siècle, à Arras, en septembre 1303, est liée au paiement de la solde, et non à l'obligation de servir ${ }^{6}$. Il n'y eut donc pas à proprement parler de mutinerie. Quant aux désertions (il serait d'ailleurs préférable de parler de départs non autorisés), il dut y en avoir comme il y en eut dans toutes les armées à toutes les époques. Mais, à notre connaissance, elles ne prirent jamais une ampleur susceptible de compromettre le déroulement des opérations. À cet égard, un seul épisode fut réellement préoccupant, mais il se situe au début de la période et dans un contexte bien particulier: après la capitulation de l'armée croisée, en avril 1250, la plupart des barons et chevaliers survivants, y compris les deux frères du roi, Alphonse de Poitiers et Charles d'Anjou, choisirent de rentrer en France au lieu de suivre Saint Louis en Syrie. Lors du conseil au cours duquel le roi demanda aux barons d'exprimer leur avis, Joinville fut un des seuls à conseiller au roi de rester; les autres hauts hommes le lui reprochèrent d'ailleurs, parfois violemment ${ }^{7}$.

${ }^{4}$ Tout au plus les uns et les autres mettaient-ils en avant le nombre des ennemis.

${ }_{6}^{5}$ Primat traduit par Jean du Vignay, RHGF, t. XXIII, p. 40.

${ }^{6}$ Sur cette révolte des soudoyers, Frantz Funck-Brentano, Les origines de la guerre de Cent ans. Philippe le Bel en Flandre, Paris, 1896, p. 455.

${ }^{7}$ Joinville, Vie de Saint Louis, éd. Jacques Monfrin, Paris, 1995, § 426-427. 
Il semble par conséquent acquis, que, eu égard aux contraintes logistiques et notamment aux impératifs du ravitaillement, qui limitaient de facto le nombre maximal de combattants qu'il était possible de rassembler, jamais le roi de France, même au pire moment, n'a connu de difficultés à mettre sur pied une armée suffisamment nombreuse - le nombre, bien entendu, n'étant qu'une des conditions du succès. En d'autres termes, on peut convenir que, de manière générale, la noblesse a répondu favorablement aux demandes du gouvernement royal, en ce sens minimal que celui-ci n'a jamais manqué de combattants.

En gardant à l'esprit ces prémisses, il reste à déterminer les raisons qui ont poussé les nobles du royaume à accepter de risquer leur vie dans des guerres parfois lointaines, dont le coût humain était potentiellement très élevé, et alors même que le royaume n'était généralement pas en danger.

On pourrait imaginer que cette question n'est pertinente que pour un Européen né dans le dernier tiers du $\mathrm{XX}^{\mathrm{e}}$ siècle, dans un pays, la France, qui n'a pas connu de conflit important depuis plusieurs décennies et où la conscription a cédé la place à une armée professionnelle dont les objectifs déclarés sont, désormais, d'ordre humanitaire. Il y a encore cinquante ou soixante ans, il en allait tout autrement. Il suffisait à chaque Français de consulter le livret militaire qu'on lui avait remis à l'issue d'un temps de service qui nous semble extravagant (deux ans), pour savoir où il devait se trouver, un, deux, trois jours après la mobilisation générale. Encore pour les Français nés dans les années 1920, la guerre était une perspective tout à fait possible, voire probable.

Mutatis mutandis, il en allait de même pour un noble de la fin du XIII ${ }^{\mathrm{e}}$ siècle. La guerre était pour lui une activité naturelle, à laquelle toute son éducation l'avait préparé. C'était en quelque sorte sa raison de vivre, et éventuellement, du coup, une raison de mourir. Dans un tel contexte, la prise de risques qu'entraînait la participation à une campagne militaire était considérée comme incompressible. La guerre faisait partie de «l'état de noblesse », et Philippe Contamine a montré qu'aux XIV et $\mathrm{XV}^{\mathrm{e}}$ s., une des idées fondamentales sur lesquelles reposait cet «état de noblesse » était justement la notion de «service»: martelée par le gouvernement royal, l'idée de service finit par devenir un lieu commun de la propagande officielle ${ }^{8}$.

Pour la fin du XIII ${ }^{\mathrm{e}}$ s., un nombre varié d'exemples permettrait cependant de remettre en cause ou, plus exactement, de nuancer, cette idée que la noblesse avait massivement vocation à servir le roi. Si, de manière générale, les derniers Capétiens n'ont jamais connu de difficultés à lever leurs armées, cela ne signifie pas que l'ensemble de la noblesse ait participé volontiers à l'effort de guerre. Saint Louis eut bien du mal à convaincre ses barons de le suivre dans ses deux expéditions. Devant le rendement décevant des convocations qu'il avait lancées en vue de la campagne contre le comte de Foix (1272), Philippe III prit des mesures sévères à l'encontre de ceux qui avaient négligé d'y obéir. Quelques décennies plus tard, il fallut à Philippe le Bel toutes les ressources d'une propagande particulièrement inventive pour faire triompher le principe du service militaire obligatoire pour l'ensemble de la noblesse puis, avec l'avènement de l'arrière-ban, pour tous les sujets du royaume. Au moment même où, avant comme après Courtrai, la chancellerie royale justifiait la poli-

\footnotetext{
${ }^{8}$ Philippe Contamine, «Noblesse et service : l'idée et la réalité dans la France de la fin du Moyen Âge », art. cit. dans la note 2.
} 
tique de Philippe le Bel en adoptant toujours le ton qu'exigeaient les circonstances ferme quand le roi était en position de force, humble dans le cas contraire - baillis et sénéchaux étaient chargés de poursuivre les nobles qui n'avaient pas obéi aux convocations pour l'armée et de les mettre à l'amende. Un véritable système répressif fut même mis sur pied pour faire payer les récalcitrants ou les contraindre à se rendre à l'armée.

En somme, le roi n'avait généralement pas de mal à mettre sur pied une armée suffisamment nombreuse; mais le vivier dans lequel il pouvait puiser ne s'identifiait pas à l'ensemble de la noblesse. Il y avait des réfractaires et, plus encore sans doute, des réticents. S'interroger sur les motivations de ceux qui ont servi nécessite donc également de prendre en compte celles des nobles qui ont ou auraient préféré demeurer chez eux, celles-ci relativisant fatalement celles-là.

De ce fait, parmi toutes les raisons possibles pour lesquelles un noble, chevalier ou écuyer, pouvait servir dans l'armée royale, il faut faire une place particulière à l'obligation qui lui en était faite. De ce point de vue, la fin du XIII ${ }^{\mathrm{e}}$ siècle a vu se jouer, entre le roi et la noblesse, une partie difficile, dont Philippe le Bel est finalement sorti vainqueur, en recueillant, dans une assez large mesure, les fruits de la politique de son père. C'est en effet à Philippe III que revient le mérite d'avoir engagé l'évolution du service militaire de la noblesse. En 1272, pour mater la révolte du comte de Foix, le roi réunit son ost en faisant appel au service que lui devaient ses vassaux directs. Les grands vassaux répondirent à la convocation royale avec le contingent très limité qu'ils devaient produire; seigneurs petits et moyens firent de même. Mais, à Tours, où les vassaux des bailliages du nord du royaume devaient se concentrer, puis à Toulouse, où ceux des sénéchaussées les rejoignirent, les clercs du roi réalisèrent que, sans être négligeable, le produit de la levée féodale était simplement moyen. Commentant les listes de l'ost de Foix qu'il n'avait pas étudiées d'assez près, Ferdinand Lot ironisait sur les faibles moyens du roi de France'. En réalité, les estimations vraisemblables auxquelles on peut arriver du nombre de combattants réunis par le roi en 1272 montent à un millier de chevaliers et à des milliers d'écuyers et d'hommes d'armes. C'est une armée bien assez nombreuse qui suivit Philippe III dans le Midi. Mais, à Pamiers, d'où commencèrent les opérations contre le château de Foix, il fallut se rendre à l'évidence: les quarante jours auxquels se limitait le service obligatoire et gratuit seraient rapidement épuisés. Que ferait-on ensuite ? Certes, il était possible d'envisager la poursuite des opérations en payant les combattants, ce que faisait déjà le roi avec l'Hôtel élargi qui était le noyau de l'armée. Mais rien n'obligeait les simples vassaux à accepter de poursuivre leur service. La levée féodale n'était plus adaptée.

La réaction du gouvernement royal fut dans un premier temps de poursuivre les contrevenants. Lors du Parlement tenu par le roi en septembre 1274, fut défini le tarif des amendes qui devaient frapper ceux qui, convoqués, étaient restés chez eux ${ }^{10}$.

\footnotetext{
${ }^{9}$ Ferdinand Lot, L'art militaire et les armées au Moyen Âge, en Europe et dans le ProcheOrient, Paris, 1946, 2 t., t. 1, p. 237.

${ }^{10}$ L'original ne semble pas avoir été conservé; le texte en a été transmis par Pierre d'Étampes, garde du Trésor des chartes, dans le BnF, lat. 10932, fol. 30 et publié à de nombreuses reprises, notamment dans les Ordonnances des rois de France, t. XI, p. 351 ; voyez
} 
Mais, dès l'ost de Sauveterre de 1276, la politique royale changea d'orientation. Comme il s'agissait d'une expédition destinée à sortir des frontières du royaume (l'objectif en était la Castille d'Alphonse X) et que le royaume n'était nullement en péril, il était difficile d'exiger le service féodal. Autant qu'on peut le savoir, Philippe III le fit cependant, avec une modification de taille : tous les combattants, semble-til, furent soldés, et beaucoup, sans doute, dès le premier jour.

L'obligation de service, en théorie dûment limitée, était donc en passe d'être considérablement étendue. Le gouvernement royal choisissait de n'en retenir que le principe, sans les limitations, moyennant cependant le versement de gages. Désormais, les vassaux du roi étaient tenus de le servir dans ses guerres, quelles qu'elles fussent, sans limitation de durée, mais en recevant systématiquement une solde.

Dans les sénéchaussées, l'évolution alla plus loin. Dans le Midi, les obligations militaires à l'égard du roi en tant que seigneur étaient moindres que dans le nord, où la main des baillis était plus pesante; moins bien définies, elles étaient aussi plus limitées. Néanmoins, à la faveur d'une situation troublée (l'occupation de la Navarre, en 1275, la guerre avec la Castille, l'année suivante, puis avec l'Aragon, à partir de 1282), les sénéchaux du Midi, et particulièrement celui de Toulouse, prirent l'habitude de convoquer régulièrement, presque annuellement, les nobles de leur circonscription. Bientôt, tous les nobles furent considérés comme devant le service, avec d'autant plus de facilité que, pour le coup, les frontières du royaume dans cette région étaient véritablement menacées. La distinction entre les vassaux directs du roi en tant que comte de Toulouse et les vassaux indirects tendit ainsi à s'effacer: tous, parce qu'ils étaient nobles, étaient désormais redevables du service à l'égard du roi, parce qu'il était le souverain.

Sous Philippe III, l'évolution fut donc double : le paiement de gages était accepté ; l'obligation de servir commençait à s'étendre à l'ensemble de la noblesse. Il revint à Philippe le Bel d'achever l'évolution. Il le fit en deux étapes qui dépendirent étroitement des circonstances. À l'occasion des guerres d'Aquitaine avec le roi d'Angleterre (1294-1297), le roi put généraliser le principe qui s'était imposé dans les sénéchaussées méridionales à la fin du règne de son père, le service obligatoire pour tous les nobles. Dans un contexte globalement victorieux et donc favorable, cette évolution fut étendue à l'ensemble des circonscriptions du domaine. À partir de 1297, le roi s'engagea dans une politique périlleuse en Flandre. D'abord couronnée de succès, elle s'enlisa après le désastre de Courtrai : deux années très difficiles (1302-1304) virent l'avènement de la notion d'arrière-ban. De la même façon que, victorieux en Aquitaine, le roi avait pu faire accepter l'extension du service à tous les nobles, de même, défait en Flandre, il put s'appuyer sur une propagande résolument inventive et délibérément dramatique pour imposer la généralisation du service militaire à tous les habitants du royaume.

Voilà, résumée en quelques lignes, l'évolution globale qui caractérisa le service militaire à la fin du XIII ${ }^{\mathrm{e}}$ siècle. Il ne semble pas avoir beaucoup bougé jusqu'aux premiers temps de la guerre de Cent ans ${ }^{11}$. Pour le sujet qui nous retient ici,

également l'analyse de Charles-V. Langlois, Le règne de Philippe III le Hardi, Paris, 1887, Appendices, Catalogue des mandements, n`33, p. 391.

${ }^{11}$ Philippe Contamine, Guerre, État et Société à la fin du Moyen Âge. Étude sur les armées des rois de France, 1337-1494, Paris et La Haye, Mouton, 1972 (Civilisations et sociétés, 24), 
l'étude des raisons pour lesquelles les nobles servaient dans les armées royales, il est certain que c'est un des éléments centraux qu'il faut prendre en compte. Les nobles servaient parce qu'ils étaient dans l'obligation de le faire.

On pourrait objecter, cependant, que, sur les dizaines de milliers de nobles que comptait le royaume, tous ne servirent pas, pas même une fois; tous, même, certainement, ne manifestèrent aucune velléité de le faire. À cette objection il faut faire deux réponses. La première est que, comme on l'a rappelé pour commencer, le roi n'avait pas de mal à constituer une armée suffisamment nombreuse avec les nobles volontaires. Le roi disposait d'un véritable vivier de chevaliers qui lui étaient directement attachés, non seulement parce qu'il était le seigneur de leurs fiefs, mais parce qu'ils tenaient de lui un fief-rente, c'est-à-dire une pension pour laquelle ils prêtaient hommage et en échange de laquelle ils devaient le service ${ }^{12}$.

Les délais de réunion des armées sont d'ailleurs remarquablement courts. L'armée vaincue à Courtrai fut rassemblée en quelques semaines par le comte d'Artois : chargé de venger la révolte connue sous le nom de «Matines de Bruges » (18 mai 1302), Robert d'Artois entra en campagne à la fin du mois de juin, soit un mois environ après que le principe de l'intervention en eût été décidé. Il ne devait pas avoir sous ses ordres une armée très nombreuse (sans doute comptait-elle quelques milliers de gens d'armes), mais elle parut suffisante à ce chef militaire très expérimenté et elle était, dans le dispositif imaginé par Philippe le Bel, le fer de lance de l'ost royal qui devait se réunir au début du mois d'août. À plusieurs reprises auparavant, c'était une petite armée de ce type qui avait fait campagne, et qui avait suffi, par exemple, au début de l'année 1300, sous le commandement de Charles de Valois, à subjuguer le comté de Flandre; du coup, les convocations beaucoup plus étendues qui avaient été lancées dans tout le royaume pour le printemps 1300 furent annulées. Au printemps 1302, l'idée de Philippe le Bel était certainement de rééditer la manœuvre. Les hommes d'armes qui composaient la petite armée de Charles de Valois en 1300 ou celle de Robert d'Artois en 1302 formaient, en quelque sorte, «l'armée d'active» du roi. À l'automne, alors même qu'il venait de connaître sa première grande défaite militaire, Philippe le Bel n'eut aucun mal à faire venir des milliers de chevaliers et d'écuyers de l'ensemble du royaume, dans des délais relativement brefs, mais beaucoup plus longs que pour la petite armée battue à Courtrai. Si ce grand ost de l'automne fut licencié sans avoir rien tenté, c'est qu'il y manquait les cadres et les meilleurs combattants, tombés nombreux à Courtrai. Pour le sujet qui nous retient ici, l'important est que le roi put compter, en ces circonstances catastrophiques, sur sa noblesse ; qu'elle n'ait été ni très enthousiaste ni très préparée à l'idée de combattre est un autre problème.

En appeler à la bonne volonté de la noblesse suffisait donc à constituer une armée suffisante. Était-il besoin alors de mettre en œuvre une politique plus coercitive ? Cela ne faisait pas de doute dans l'esprit des rois, de leurs conseillers et de leurs agents, et c'est la deuxième réponse qu'il faut apporter à l'objection soulevée plus haut. Parmi leurs attributions, baillis et sénéchaux étaient en effet chargés de conduire au point de concentration fixé par le roi le contingent de leur circonscrip-

Première partie, «Les forces militaires de la monarchie française au milieu du XIV siècle (1337-1369)», p. 3-131.

${ }^{12}$ Sur l'institution du fief rente, le livre classique de Brice D. Lyon, From Fief to Indenture, Cambridge Mass., 1957. 
tion. Assez souvent, surtout dans le Midi, ils avaient aussi à mener eux-mêmes des opérations militaires, parfois de grande envergure ${ }^{13}$. Baillis et sénéchaux étaient de fait des chefs militaires. Quelques indices permettent d'établir qu'ils ne manquaient pas de moyens de pression. Au moment où se préparait l'expédition de Foix, en mai 1272, Barthélemy, seigneur de L'Île-Bouchard, à qui Philippe III avait ordonné de porter son hommage à son favori Pierre de La Broce, mais qui, à cette date, était encore le vassal direct du roi, fut convoqué pour suivre l'armée avec un certain nombre de chevaliers. Victime d'une attaque de goutte qui le paralysait, il ne pouvait se rendre à l'ost. Il pria donc Pierre de parler au roi en sa faveur, afin qu'une lettre de répit fût envoyée au bailli. La demande que formulait Barthélemy, en des termes pressants, afin que le favori, son nouveau seigneur, intervînt auprès du roi, illustre bien l'importance du bailli dans la procédure de convocation des vassaux: manifestement, Barthélemy craignait d'être considéré comme défaillant ${ }^{14}$. Deux ans plus tard, en 1274, une guerre privée opposait deux nobles picards, Raoul de Flavy et Jean de l'Essart. Ce dernier, convoqué par les gens du roi pour l'ost de Montfaucon dirigé contre Rodolphe de Habsbourg, demanda au prévôt de Roye de lui procurer une trêve avec son adversaire, le temps qu'il serait au service du roi. Le prévôt en référa aux serviteurs du seigneur de Nesle, dont Raoul de Flavy était le vassal. «Et quant mesires Jehans vit che, il n'osa laissier por le doute le roi qu'il n'alast u serviche le roy a Montfaucon $»^{15}$. Le doute le roi : on ne prenait pas à la légère les ordres du roi, et les baillis semblent avoir été particulièrement craints. Leurs mandements, de ce fait, étaient respectés. Pour l'ost de Foix de 1272, il est vrai que de nombreux vassaux ne répondirent pas à la convocation royale ; mais il faut prendre note du zèle que bien d'autres mirent à le faire. Souvent, certes, les personnes convoquées tentèrent de minimiser ou, dans certains cas, de nier leurs obligations ; mais ils firent malgré tout le déplacement, au lieu d'ignorer la convocation qui leur avait été adressée. C'est que, la plupart du temps, ils ne refusaient pas de servir. Ils refusaient de servir gratuitement. Après l'ost de Foix, Gui de Lévis, seigneur de Mirepoix, maréchal d'Albigeois, se pourvut devant le Parlement. Il prétendait que lui seul devait le service au roi, avec neuf autres chevaliers, et que ses vassaux ne devaient rien de la sorte. Il fut débouté ${ }^{16}$. Mais rien ne dit que, dans cette batailleuse famille des Lévis, Gui ait été réticent à servir dans l'armée royale ; simplement, il ne voulait pas y être contraint, et encore moins gratuitement, et il ne voulait pas non plus que ses vassaux, simples arrière-vassaux du roi, soient tenus au service militaire envers un autre que lui-même. L'évolution amorcée avec l'ost de Sauveterre répondit à ces réticences, en généralisant le versement des gages.

${ }^{13}$ Les sénéchaux de Toulouse se trouvèrent ainsi souvent en première ligne. Charles-Victor Langlois, l'historien de Philippe le Hardi, voyait en Eustache de Beaumarchais, sénéchal de Toulouse et, un temps, gouverneur de Navarre, le «Du Guesclin du règne » (Le règne de Philippe III le Hardi, Paris, 1887, p. 43).

${ }^{14}$ Sur cette affaire, je me permets de renvoyer à un article à paraitre dans le Journal des Savants, «Pierre de La Broce, seigneur féodal, et le service militaire sous Philippe III. L'ost de Sauveterre (1276)».

${ }^{15}$ E. Boutaric, Actes du Parlement de Paris, $1^{\text {ere }}$ série, De l'an 1254 à l'an 1328, 2 vol., Paris, 1863-1867, nº1987 B (d'après Arch. nat., J 1028, n¹4).

${ }^{16}$ E. Boutaric, Actes du Parlement de Paris, ${ }^{\circ} 1869$ (Toussaint 1272) ; cf. dom Devic et dom Vaissète, Histoire générale du Languedoc, nouv. éd. par Auguste Molinier, t. IX, p. 18. 
Certains, cependant, refusaient probablement la perspective de servir le roi les armes à la main, même en échange de gages. On les aperçoit mieux sous le règne suivant. Après l'expédition de Foix, Philippe III avait ordonné de faire payer ceux qui n'avaient pas obéi à sa convocation; faute de documents, on ne sait pas ce qu'il en fut en réalité. Sous Philippe le Bel, la documentation disponible est beaucoup plus nombreuse. Les comptes des bailliages et des sénéchaussées montrent que les mandements par lesquels le roi ordonnait à ses représentants de poursuivre les réfractaires et de les mettre à l'amende étaient exécutés. Ils donnent les quelques jalons qui suffisent à attester la persistance d'une pratique. En 1297, le roi réunit une armée destinée à envahir le comté de Flandre. Les contingents des bailliages furent convoqués. Parmi les extraits du rôle des bailliages de France pour l'Ascension 1298 se trouve cette ligne de dépense : Pro parum computatis pro nobilibus compellendis ut irent in exercitum, $20 \mathrm{~s}^{17}$. Il fallait donc exercer une certaine pression sur les nobles pour qu'ils se rendent à l'ost. En 1299, sur l'ordre du roi, Gérard de Condé, un de ses clercs, dressa un compte récapitulatif des diverses subventions levées dans le bailliage de Caux. Seule une petite partie en a été conservée ${ }^{18}$. Mais le sous-titre de ce travail mérite d'être considéré avec attention ${ }^{19}$. Parmi les multiples taxes auxquelles avait donné naissance l'ingéniosité fiscale des conseillers de Philippe le Bel, se trouvent en effet des financie nobilium pro excercitu (sic) Flandrensi et quorumdam grosseriorum 1000 libras habentium. L'estimation de leur rapport par Gérard de Condé est perdue. Peu importe : l'essentiel est que, au plus tard en 1297, il a été imposé aux nobles de racheter le service d'ost. C'est donc bien qu'il était obligatoire, et qu'à cette date, le système de compensation financière était déjà en place : c'était au fond, certes, celui qu'avait élaboré Philippe III après l'ost de Foix, en imposant et en tarifant une amende à ceux qui ne s'étaient pas rendus à l'ost. La seule différence, mais elle est de taille, c'est que ce n'étaient plus seulement les vassaux du roi mais tous les nobles qui étaient concernés. À son tour, le compte du bailliage de Tours pour la Toussaint 1299 cite parmi ses recettes des Financie nobiliorum qui non fuerunt in exercitu Flandrie ${ }^{20}$. Les nobles : ni les vassaux, ni les chevaliers, mais les nobles. La liste des douze contrevenants indique d'ailleurs des chevaliers (qualifiés de dominus) et des écuyers (sans cette mention), mais rien ne dit qu'ils étaient les vassaux du roi. L'amende qu'ils durent payer était peu élevée, et elle était variable (de 4 à 20 1., avec une moyenne de 101 .); il faut penser que c'est

${ }^{17}$ Robert Fawtier, avec le concours de François Maillard, Comptes royaux (1285-1314), volume I, Comptes généraux, Paris, Imprimerie Nationale, 1953 ; volume II, Comptes particuliers et comptes spéciaux ou extraordinaires, Paris, Imprimerie Nationale, 1954 ; volume III, Introduction. Appendice. Suppléments. Indices, Paris, Imprimerie Nationale, 1956 (Recueil des historiens de France, Documents financiers, tome III, trois volumes) ; la numérotation est continue dans les trois volumes. ; désormais, Comptes royaux, $\mathrm{n}^{\circ} 345$.

${ }^{18}$ Comptes royaux, $\mathrm{n}^{\circ} 21231-21238$.

${ }^{19}$ Apprisia facta, de mandato domini regis, per dominum Gerardum de Condeto, presbiterum, et Droconem Perigrini super valore centesime, quinquagesime, secunde quinquagesime sive novissime et muttualis subventionis, necnon auxilii et obolorum pro facto maris, financiarumque nobilium pro excercitu Flandrensi et quorumdam grosseriorum 1000 libras habentium et assisie domini regis, que malatolta dicitur, in ballivia Caletensi et ressortis ejusdem, anno Domini 1299.

${ }^{20}$ Comptes royaux, ${ }^{\circ} 2976-2988$. 
l'importance de leur patrimoine qui déterminait le montant de leur «finance ». Même d'un faible montant, celle-ci était payée avec réticence : le même compte du bailliage de Tours enregistre la dépense de 91.14 s. pour envoyer les sergents exiger les «finances» des nobles qui ne s'étaient pas rendus à l'ost ${ }^{21}$.

Après Courtrai, le pouvoir du roi se trouva brutalement déstabilisé. Philippe le Bel comprit le risque. Il montra à la fois ce mélange de fermeté et de compromis qui est la marque de ces années difficiles. Comme l'avait fait son père après les osts de Foix et de Navarre, trente ans auparavant, une des premières mesures que prit le roi à son retour des frontières de Flandre fut de faire lever des amendes sur ceux, nobles et non-nobles, qui, dotés des revenus suffisants, n'avaient pas accompli le service d'ost ; cette mesure devait être facilitée par l'inscription, sur les quittances délivrées à chaque chevalier et écuyer ayant pris part à la campagne, du bailliage d'origine : les clercs du roi durent en tirer des listes par circonscription, qui permirent ensuite de rechercher les contrevenants. Le 18 octobre 1302, le roi mandait à Jean de Dijon et au bailli de Mâcon de lever des amendes sur les nobles ayant au moins $40 \mathrm{l}$. de revenu et sur tous, nobles ou non-nobles, ayant $300 \mathrm{l}$. de biens, qui n'auraient pas accompli le service d'ost ${ }^{22}$. Le pragmatisme, cependant, l'emportait à l'occasion : à la prière du comte de Rodez, un puissant vassal qu'il fallait ménager d'autant plus qu'il s'était rendu, lui, à l'ost, Philippe le Bel, le 5 octobre 1302, remit à tous les feudataires de celui-ci les amendes qu'ils avaient pu encourir pour ne pas être venus à l'ost de Flandre - même l'arrière-ban souffrait quelques aménagements ${ }^{23}$. Surtout, comme les nobles s'étaient rendus en masse à l'armée et que celleci n'avait pas engagé le combat, il était délicat pour le roi de se montrer trop dur: tout restait à faire contre les Flamands et il ne fallait pas compromettre les campagnes futures ${ }^{24}$.

On pourrait solliciter d'autres exemples encore. Ceux-ci suffisent à montrer que les représentants du roi savaient se faire les relais efficaces de ses ordres. À la façon de la moderne gendarmerie militaire, baillis et sénéchaux veillaient à ce que les convocations militaires fussent obéies. Ils étaient certainement responsables, personnellement, du bon déroulement des opérations de mobilisation : ils avaient donc tout intérêt à se montrer zélés. Décidément, si les nobles servaient, c'est bien d'abord parce qu'ils y étaient tenus : c'est une des grandes avancées qu'a connues le pouvoir royal sous les règnes de Philippe III et de son fils.

Néanmoins, l'obligation ne valait que pour certaines campagnes menées par l'armée royale. À trois reprises dans la période envisagée, le roi prit la tête d'une croisade : Saint Louis, à deux reprises, en 1248 et en 1270 ; Philippe III, en 1285.

\footnotetext{
${ }^{21}$ Comptes royaux, $\mathrm{n}^{\circ} 3210$ : Pro expensis quorumdam servientium ballivie, missorum huc et illuc per balliviam, pro compellendo nobiles dicte ballivie ad solvendum financias ab ipsis debitas pro eo quod non fuerunt in exercitu Flandrensi [...].

${ }^{22}$ Arch. nat., JJ 36, fol. 9 v, n² 28 ; éd. La Roque, Traité du ban et de l'arrière-ban, p. 141 ; la mesure fut rappelée quelques jours plus tard, le 9 novembre : voyez Ordonnances des rois de France, t. I, p. 350-351.

${ }^{23}$ Arch. dép. Tarn-et-Garonne, A 316, fol. $36^{\mathrm{v}}$.

${ }^{24}$ Le roi s'avisa brutalement des abus de ses agents : le 22 octobre 1302, Jean de Gonesse, chanoine de Chartres, et Guillaume de Nogaret, chevalier, furent chargés de se rendre dans le bailliage de Senlis pour y enquêter et y mettre bon ordre (JJ 36, fol.12, n 38 et 39 ; cf. aussi $\left.\mathrm{n}^{\circ} 40\right)$.
} 
Rien, à la vérité, ne semble distinguer les armées réunies en ces circonstances de celles dont nous avons étudié plus haut les modalités de la réunion. Ce sont, pour ce qu'on peut en savoir, les mêmes combattants, la même organisation, le même ordre de grandeur des effectifs rassemblés. Néanmoins, l'obligation féodale ne pouvait jouer puisque la croisade, qu'on ne pouvait entreprendre qu'à la suite d'un vœu délibéré, échappait au cadre bien délimité des obligations vassaliques. D'autres expéditions conduites ou patronnées par les rois se trouvèrent dans ce cas. En 1265, Charles d'Anjou mit sur pied une expédition, bénie par l'Église et soutenue, dans des proportions mal connues, par son frère Saint Louis. Sa victoire, l'année suivante, à Bénévent, lui assura les clés du royaume de Sicile. En 1282, Philippe III envoya une petite armée combattre au nom du pape Martin IV dans une région rétive à l'autorité pontificale, la Romagne. À la fin de cette même année, une expédition conduite par les comtes d'Alençon et d'Artois, neveux de Charles d'Anjou, prit à son tour le chemin de l'Italie, cette fois pour porter secours à ce dernier aux prises avec la révolte de la Sicile, dite des «Vêpres Siciliennes ».

Il y eut donc des cas, nombreux, où, bien que le principe du service militaire ne pût jouer, une partie de la noblesse servait volontiers. Le goût des armes devait jouer. On s'expliquerait mal la carrière du comte d'Artois sans évoquer une attirance poussée pour la vie des camps. De 1270 à sa mort en 1302, Robert d'Artois ne cessa de mener campagne: croisade de Tunis (1270), séjour en Toscane au service de Charles d'Anjou (1274), expédition de Navarre (1276), expédition de secours en Italie et régence du royaume de Naples (1282-1291), campagnes d'Aquitaine (12961297) et de Flandre (1297), ponctuées par deux victoires importantes (Bonnegarde et Furnes) ; une dernière et fatale campagne, en juin-juillet 1302, fut précédée de tractations avec la papauté en vue d'une nouvelle expédition italienne, dont Charles de Valois prit finalement la tête. Entre deux campagnes, le comte d'Artois fréquentait assidûment les tournois. Les comptes qu'on a conservés sont remplis de mentions de paiements faits à ses chevaliers pour les pertes occasionnées par les tournois dans lesquels ils l'avaient suivi ; mieux, Robert d'Artois fut le héros du Roman du Hem, un précieux récit en vers composé à partir du tournoi tenu dans cette petite seigneurie picarde en $1278^{25}$. Le tournoi, certes, n'était pas la guerre, mais il en était fort proche. À cet égard, on peut souscrire à l'idée que soutenait le grand historien de la dynastie angevine, Émile Léonard, qui voyait dans la volonté de fuir l'ennui l'une des motivations principales des chevaliers qui avaient suivi Charles d'Anjou ${ }^{26}$.

Ils durent être assez nombreux, ceux qui, sur le modèle du comte d'Artois, étaient assez épris du métier des armes pour ne jamais rechigner devant une nouvelle campagne ou un nouveau tournoi. Un certain «Pierres Pillart, du Ménil, chevalier de la terre de Biaumont », mal pris avec le prévôt local à propos d'une histoire de vol d'un cheval, présenta une supplique bien connue à un roi qui est sans doute Philippe

\footnotetext{
${ }^{25}$ Sarrasin. Le Roman du Hem, éd. Albert Henry, Paris, Les Belles Lettres, 1938 (Travaux de la faculté de philosophie et lettres de l'université de Bruxelles, 9).

${ }^{26}$ Émile G. Léonard, Les Angevins de Naples, Paris, 1954, p. 16 : «En sorte que, lorsque vient à manquer le suprême moyen de divertissement, la guerre, dernière ressource de l'homme pour redonner à la vie, en la risquant à chaque instant, une saveur qui s'évanouit aussitôt, il faut chercher autre chose ou partir vers de nouveaux cieux ». Il faut préciser que, quelques lignes plus haut, É. Léonard range implicitement les chevaliers du XIII ${ }^{\mathrm{e}}$ s. parmi les «populations primitives».
} 
III $^{27}$. Pour fléchir le roi en sa faveur, Pierre Pillart mettait en avant ses états de service : «Je sui vous et vos anceseus en l'alé que en n'ala a Damieste, et que en ala en Sesile, et au siege a Marselles, et au siege de Tunes. En toz ces lius, trovarant chevaliers et bones gens qui m'aront veu et quonneu mes euvres ». Qu'un chevalier totalement inconnu par ailleurs, de toute façon d'extraction parfaitement obscure, et dont seul le hasard nous a préservé le nom, ait ainsi pris part à la croisade d'Égypte (1248-1250), au siège de Marseille par Charles d'Anjou (1262), à la croisade de Sicile menée par ce dernier (1265-1266) et à l'expédition de Tunis (1270) laisse penser qu'il n'avait pas de réticence particulière à combattre. On est en droit de penser qu'il ne devait pas être seul de son espèce.

Pour toutes ces expéditions, les combattants étaient payés, soit sous forme de gages soit sous forme de «convenances». Ces contrats passés avant le départ de l'expédition entre le roi et tel baron ou tel chevalier précisaient les engagements des deux parties. Est-ce pour autant l'attrait de la solde qui poussait les gens d'armes à se mettre sous les ordres d'un chef d'armée ? C'est évidemment un problème difficile à résoudre, et il se pose également pour toutes les expéditions où des gages étaient versés. Bien entendu, ce ne devait pas être dans tous les cas un paramètre négligeable. Mais encore faudrait-il être en mesure de déterminer l'importance relative de la solde ramenée aux ressources d'un noble moyen ${ }^{28}$. Était-ce une entrée pécuniaire espérée, ou seulement, au contraire, le moyen de couvrir les frais engagés pour une expédition coûteuse (chevaux, armes, équipement, nourriture) - sans même qu'on y inclue les risques personnels ? Bien souvent, en réalité, le bénéfice en matière financière devait être vraiment minime, et on incline à penser que la guerre n'était pas, en règle générale, une occasion de s'enrichir. Bien au contraire, les chefs de l'armée, hauts barons et souvent parents du roi, servaient fréquemment à leurs frais ; chevaliers et écuyers du rang pouvaient attendre dix ans le versement de leurs gages $^{29}$. Seuls quelques combattants particulièrement valeureux pouvaient espérer tirer bénéfice de la guerre et encore était-ce la plupart du temps de manière indirecte, sous la forme de récompenses attribuées par le souverain. À la faveur des guerres de Philippe le Bel, on vit ainsi de belles carrières se faire. Un simple arbalétrier de l'Hôtel pouvait terminer chevalier du roi, à la tête d'un patrimoine fort opulent ${ }^{30}$. En règle générale, cependant, ce n'est pas avec sa solde qu'on revenait enrichi d'une

${ }^{27}$ Ce remarquable petit dossier a été édité deux fois à un demi-siècle de distance : Louis Douët d'Arcq, "Supplique d'un chevalier contre un déni de justice ; pièces du XIII" siècle », Bibliothèque de l'École des Chartes, 9 (1848), p. 405-411; Élie Berger, «Requête adressée au roi de France par un vétéran des armées de Saint Louis et de Charles d'Anjou », Études d'histoire du Moyen âge offertes à Gabriel Monod, Paris, 1896, p. 343-350.

${ }^{28}$ Voyez le tableau des gages établi par Philippe Contamine, Guerre, État et société, op. cit., Annexe VI, p. 619-636.

${ }^{29}$ En février 1309, Philippe le Bel ordonna à Jean de Crépy, son clerc, chargé de la perception de la décime et des annates, de payer à Humbert de Romans, chevalier, ses gages et ceux de sa compagnie pendant la guerre d'Aquitaine ; les opérations actives en Aquitaine ayant cessé en 1297, il est probable que ces gages étaient dus depuis plus de dix ans (BNF, fr. 25697, $\left.\mathrm{n}^{\circ} 44\right)$.

${ }^{30}$ C'est le cas d'Ourri l'Allemand, dont la carrière peut être suivie de 1285 à 1316 : voyez la thèse citée dans la note 1 , aux p. 687-688. 
guerre, même victorieuse. Joinville perdit beaucoup lors de la croisade d'Égypte, au point de refuser, vingt ans plus tard, de reprendre la croix ${ }^{31}$.

La solde, pourtant, n'était pas la seule récompense qu'un chevalier optimiste ou assez chanceux pour survivre aux combats pouvait attendre. L'appât d'un gain plus substantiel devait jouer également. Une entrée dans la faveur royale s'accompagnait dans la plupart des cas de dons plus ou moins élevés. Mais il y avait mieux. La guerre pouvait changer un destin. À cet égard, les exemples venaient de loin. La première croisade avait vu quelques grands seigneurs devenir rois de Jérusalem, princes d'Antioche ou comtes d'Édesse. De façon encore plus frappante, les Hauteville, d'une famille pour le moins indistincte du Cotentin, s'étaient élevés jusqu'au trône de Sicile et des mariages prestigieux les avaient apparentés à la famille capétienne. Mais l'épisode que, dans la seconde moitié du XIII ${ }^{\mathrm{e}}$ siècle, tous les nobles ambitieux devaient garder en mémoire, était certainement la prise de Constantinople et les événements qui l'avaient suivie. De simples seigneurs champenois ou bourguignons avaient été promus du jour au lendemain princes de Morée ou ducs d'Athènes. Ils avaient gardé avec la France des liens étroits. Othon de La Roche, duc d'Athènes, s'était retiré pour ses vieux jours dans ses terres patrimoniales. Les empereurs latins eux-mêmes demeuraient comtes de Namur ou sires de Courtenay. Encore à l'extrême fin du XIII ${ }^{\mathrm{e}}$ s. et au début du siècle suivant, des seigneurs français se cherchaient un avenir en Grèce ou en Terre sainte. Un Gautier, comte de Brienne, devint duc d'Athènes, ce qui permit à son fils, le connétable de France, de porter, en plein $\mathrm{XIV}^{\mathrm{e}}$ s., un titre qui devait avoir une saveur quelque peu archaïque ${ }^{32}$.

La perspective de conquérir de vastes terres ne devait donc pas être étrangère à beaucoup de nobles, en particulier à ceux qui, en 1265, se joignirent à Charles d'Anjou. Ils en furent d'ailleurs récompensés, puisque le conquérant ne leur ménagea pas ses faveurs, surtout quand la descente italienne de Conradin, en 1268, eut révélé la fragilité de son assise auprès de la noblesse locale ${ }^{33}$. En 1285 , il n'est pas impossible que les nobles qui décidèrent d'accompagner Philippe III dans son expédition contre le roi d'Aragon aient partagé des arrière-pensées similaires. Au demeurant, le but de l'expédition était tout à fait clair : il s'agissait de conquérir le royaume d'Aragon, que le pape avait octroyé au second fils du roi de France, Charles de Valois. Aux dires de Desclot, le chroniqueur catalan contemporain dont le témoignage est le plus souvent sujet à caution quand il concerne les Français, Charles de Valois aurait établi par avance un sénéchal pour ses terres d'Aragon encore à conquérir et le partage des fiefs aurait commencé avant même le passage des Pyrénées ${ }^{34}$. L'échec de l'expédition ne permit pas la réédition des exploits accomplis par Charles d'Anjou deux décennies auparavant, mais il est probable que c'est ce qu'avaient en vue les croisés de 1285.

À ces perspectives de lendemains enchanteurs, s'ajoutait bien entendu la quête de la gloire. Pendant un des épisodes difficiles de la campagne d'Égypte, en

\footnotetext{
${ }^{31}$ Joinville, Vie de Saint Louis, éd. cit., notamment $\$ 735$.

${ }^{32}$ Sur tout le paragraphe qui précède, Jean Longnon, L'Empire latin de Constantinople et la principauté de Morée, Paris, 1949.

${ }^{33}$ É. Léonard, Les Angevins de Naples, op. cit., notamment aux p. 80-82 ; et aussi, naturellement, Paul Durrieu, Les archives angevines de Naples. Étude sur les registres du roi Charles $I^{e r}$, Paris, 2 t. 1887.

${ }^{34}$ Joseph Petit, Charles de Valois, Paris, 1900, p. 8.
} 
1250, le comte de Soissons dit à Joinville: "Seneschal, lessons huer cette chiennaille, que, par la quoife Dieu ! - ainsi comme il juroit - encore en parlerons nous entre vous et moy de ceste journée ès chambres des dames $»^{35}$. Le désir de se couvrir de gloire n'était sans doute pas le fait exclusif du comte de Soissons. En dépit des efforts des chefs de guerre, la discipline était fort difficile à garder dans les rangs de l'armée française, tant était forte la soif de se mettre en avant, au propre comme au figuré. Même si l'on fait la part des topoi qui structurent tout récit de défaite, il ne fait pas de doute que l'individualisme chevaleresque, qu'un Joinville, d'ailleurs, ne condamne pas, pas plus que certains chroniqueurs ecclésiastiques, fut la cause de l'échec qui marqua, en règle générale, les entreprises militaires françaises de la période $^{36}$. Quoi qu'il en soit, on peut en tirer l'idée que la quête de gloire ne comptait pas pour rien dans l'engagement des nobles dans les armées royales.

Cette constatation n'est pas valable seulement pour les expéditions lointaines. On pouvait se couvrir de gloire en Flandre et en Aquitaine aussi bien qu'en Égypte ou en Italie, et sans y courir les risques liés aux pays chauds dont le climat convenait assez mal aux Français. Pour les jeunes gens, une campagne militaire, où qu'elle se déroulât, était l'occasion de montrer leur valeur et, si ce n'était pas encore fait, de recevoir la «ceinture de chevalerie » (cingulum militie). Quel adoubement avait plus de valeur que celui qui était célébré le matin d'une bataille, avant le départ de l'armée ou au retour d'une campagne ${ }^{37}$ ? C'est d'autant plus vrai, certainement, qu'à la même époque l'adoubement avait perdu de son attrait: beaucoup de nobles restaient écuyers toute leur vie, au point que Philippe le Bel, comme son exact contemporain le roi d'Angleterre Édouard Ier, devait s'en inquiéter et menacer de ses foudres ceux qui refusaient de se faire adouber. Pour certains, cependant, être adoubé, et l'être dans un contexte proprement militaire, restait, à ce qu'on peut en juger, très prestigieux; du moins pour les classes supérieures de la noblesse - rappelons que Joinville, par exemple, ne cite jamais nommément que des chevaliers. Que les rois de France et d'Angleterre aient été contraints de prendre des mesures coercitives en faveur de l'adoubement laisse penser que, en demeurant écuyers, beaucoup espéraient échapper aux obligations militaires et aux frais qui s'attachaient à la qualité chevaleresque. Si l'élite de la noblesse ne pouvait se passer du titre de chevalier, en revanche, dans leurs manoirs campagnards, les familles moins reluisantes ne se sentaient pas déshonorées de ne plus le porter. Un seigneur bourguignon qui fit compiler vers 1270 un cartulaire, Jean de Nesle - sans rapport avec la puissante famille picarde -, se fit adouber à quarante ans passés. On ne sait d'ailleurs s'il prît jamais part à une quelconque campagne militaire ${ }^{38}$.

La volonté de servir pouvait aussi procéder de la fascination exercée par un chef charismatique. Telle qu'elle se présentait en 1265, l'entreprise de Charles

\footnotetext{
${ }^{35}$ Joinville, Vie de Saint Louis, éd. cit., § 242.

${ }^{36}$ Françoise Autrand, «La déconfiture. La bataille de Poitiers (1356) à travers quelques textes français des $\mathrm{XIV}^{\mathrm{e}}$ et $\mathrm{XV}^{\mathrm{e}}$ s. », Guerre et société en France, en Angleterre et en Bourgogne, $X I V^{e}$ et $X V^{e}$ siècles, éd. Philippe Contamine, Ch. Giry-Deloison et M. H. Keen, Lille, 1991, p. $93-121$.

${ }_{37}$ À ce propos, voir Philippe Contamine, «Points de vue sur la chevalerie en France à la fin du Moyen âge », art. cit., p. 272.

${ }^{38}$ La publication de ce beau cartulaire est en cours (Musée Condé, Archives du château de Chantilly, n'XIV F; Stein 2710).
} 
d'Anjou apparaissait risquée. Le frère du roi d'Angleterre Henry III, Richard de Cornouailles, à qui on avait proposé la couronne de Sicile, s'était bien gardé de se lancer dans l'aventure. Même s'il s'agissait d'une croisade et même si le prétendant au trône de Sicile avait promis à ceux qui le suivraient une solde, il est probable que l'un des éléments déterminants a été la personnalité propre de Charles d'Anjou, que tous les contemporains disent avoir été doté d'un très fort charisme. Gilles le Muisit explique ainsi que Charles était à la tête d'un grand nombre de combattants en Italie, parce que le pape avait donné des indulgences, et etiam quia princeps erat nominatissimus et curialis, in armis expertus et virtutum floribus pre ceteris perornatus ${ }^{39}$. Il en va de même, certainement, pour le principal chef de guerre des armées françaises à l'époque, le comte d'Artois, neveu de Saint Louis. Ce que les chroniques disent de lui laisse penser que c'était une personnalité de premier plan ; par ailleurs, inlassable commandant d'armées, dans les guerres de Philippe III et de Philippe IV aussi bien que comme régent de Sicile, il avait acquis une aura qui faisait de lui le meilleur homme de guerre de son temps. De fait, jusqu'au désastre de Courtrai, il fut toujours victorieux. On ne déteste pas, généralement, servir un général chéri par la victoire. Il en allait de même de Charles de Valois, le frère de Philippe le Bel. Quant aux rois de France eux-mêmes, commandants naturels de l'armée, ils étaient dotés d'un charisme assez différent selon les cas. Saint Louis et Philippe III prirent en personne la tête de leurs armées, au point de succomber en campagne (mais de maladie), le premier à Tunis, le second à Perpignan. Les chroniques nous disent que Saint Louis veillait jalousement à exercer en personne le commandement de l'armée ; sur Philippe III, les informations manquent, mais l'historien américain William Jordan a pu supposer récemment que le fils de Saint Louis aimait le «théâtre de la guerre ", the drama of $w_{a r}{ }^{40}$. Philippe le Bel, en revanche, répugna à exercer en personne le commandement de l'armée; il y délégua généralement son cousin, Robert d'Artois, son frère, Charles de Valois, ou encore le connétable, Raoul de Clermont puis Gaucher de Châtillon. Il fallut les circonstances exceptionnelles qui suivirent la défaite et la mort du comte d'Artois à Courtrai pour que le roi se mît à la tête de l'armée et prît une part décisive à la bataille de Mons-en-Pevèle. Son courage physique n'est pas en cause : en 1285, pendant la campagne de Catalogne, âgé de seize ans environ, il avait secondé efficacement son père à la tête d'une partie de l'armée. On peut supposer que, peu soucieux de renouveler l'exemple de ses trois prédécesseurs, tous morts en campagne, il préférait rester loin de la ligne de front. Après Courtrai, néanmoins, alors même qu'il demandait à la noblesse un effort particulièrement important, il ne put faire autrement que de donner l'exemple. La situation délicate dans laquelle il se trouva au cours de la bataille de Mons-en-Pevèle fut d'ailleurs largement reprise par les chroniques qui assurèrent le passage à la postérité de l'épisode. Pour susciter l'enthousiasme ou entretenir la fidélité, le chef de l'armée n'avait pas besoin d'être victorieux : il suffisait qu'il s'exposât, et qu'il partageât les risques qu'il faisait prendre à tous : quelques décennies plus tard, peu de critiques

\footnotetext{
${ }^{39}$ Chronique et annales de Gilles le Muisit, abbé de Saint-Martin de Tournai (1272-1352), éd. H. Lemaître, Paris, 1906.

${ }^{40}$ William Chester Jordan, «The Struggle for Influence at the Court of Philip III : Pierre de la Broce and the French Aristocracy », French Historical Studies, 24 (2001), p. 439-468, à la p. 466 .
} 
s'abattirent sur le valeureux Jean le Bon - elles se concentrèrent plutôt sur la noblesse défaillante ${ }^{41}$.

Si son charisme propre ne suffisait pas à déchaîner l'enthousiasme des nobles, le roi pouvait avoir recours à des méthodes plus contournées. Ce fut le cas particulièrement de Saint Louis, qui manifesta à l'occasion une «sainte duplicité ». Au témoignage de Matthieu Paris, devant le peu d'enthousiasme des barons à prendre la croix à sa suite, Saint Louis choisit un subterfuge fort habile, en faisant coudre des croix sur les vêtements qu'il fit distribuer, mine de rien, aux chevaliers de son hôtel, à l'occasion de la fête de Noël $(1245)^{42}$. Pour sa deuxième croisade, il procéda différemment. Le 25 mars 1267, il annonça sa prise de croix, qui n'eut pas le succès escompté : Saint Louis, en effet, n'emporta l'adhésion que de ses parents les plus proches. Il fallut, à la Pentecôte suivante, le faste déployé le jour de l'adoubement de son fils Philippe, fait chevalier en compagnie de plusieurs dizaines d'autres jeunes gens, pour que la croisade prît enfin consistance ${ }^{43}$. Outre les nouveaux adoubés, qui, en ce jour solennel, pouvaient difficilement refuser de se croiser, nombreux, sans doute, furent ceux qui, saisis par l'enthousiasme du moment, se rallièrent à l'idée d'une nouvelle croisade. Par la suite, du reste, l'adoubement de l'héritier de la couronne fut intimement lié à la préparation d'une croisade : en 1284, le futur Philippe le Bel fut adoubé alors que son père préparait la «croisade d'Aragon »; en 1313, le retour vers l'Orient qui caractérise la fin du règne de Philippe le Bel fut marqué symboliquement par l'adoubement de ses trois fils et de plusieurs dizaines de chevaliers, dans un contexte que dominait la perspective d'une nouvelle croisade de grande ampleur. Pour qu'il y eût croisade, cependant, il fallait désormais que le roi déployât tout un faste, sans parfois parvenir à convaincre à tout coup. Pensons à Joinville, qui cherche, il est vrai, à justifier son propre refus d'accompagner Saint Louis en insistant sur la santé déclinante de ce dernier et un manque d'enthousiasme généralisé dans l'entourage même du roi.

Ce déclin de la croisade dans les motivations des chevaliers français à la fin du XIII ${ }^{\mathrm{e}}$ siècle mériterait d'être étudié plus attentivement, tant, tout au long de l'histoire des croisades, les Français furent certainement les plus nombreux ${ }^{44}$. L'idéal de la croisade restait vif dans la littérature chevaleresque, ou chez un Rutebeuf ; il demeurait fortement présent dans les bulles de la chancellerie pontificale. Mais, dans

\footnotetext{
${ }^{41}$ Voyez notamment l'art. cit. plus haut de Françoise Autrand, « La déconfiture. La bataille de Poitiers (1356) à travers quelques textes français des $\mathrm{XIV}^{\mathrm{e}}$ et $\mathrm{XV}^{\mathrm{e}}$ s. », et Élisabeth Carpentier, «L'historiographie de la bataille de Poitiers au quatorzième siècle », Revue historique, 253 (1980), p. 21-58.

${ }^{42}$ Jean Richard, Saint Louis, Paris, 1983, p. 179.

${ }^{43}$ H. Géraud, Chronique latine de Guillaume de Nangis de 1113 à 1300, t. I, Paris, 1843 , p. 232 : Ludovicus, rex Francie, in festo Pentecostes, fecit Philippum primogenitum suum et Robertum comitem Attrebatensem, nepotem suum, novos milites, cum pluribus aliis apud Parisius. Ubi tanto fuit laetitiae solemnitas, quod populus civitatis Parisius ab omni opere vacans, solummodo laetitiae et exultationi intentus, per octo dies et amplius, civitate per totum cortinis pannorum varii coloris et ornamentis pretiosis mirabiliter palliata, solemnitatem protenderent.

${ }^{44}$ Voyez a contrario, sur la place très réduite de la croisade dans la société anglaise, les éclairantes conclusions auxquelles parvient Simon Lloyd, English Society and the Crusade, Oxford, 1988, p. 244-247.
} 
la plupart des esprits, la croisade s'éloignait définitivement. De manière générale, l'insuccès de Tunis acheva de confirmer ce déclin. Pourtant, si l'on excepte la solitude relative de Saint Louis en Syrie de 1250 à 1254, la croisade n'a pas été l'occasion pour la noblesse de se détacher du service du roi. Au contraire, même, le roi de France était bien le seul souverain européen à pouvoir rassembler une vingtaine de milliers d'hommes pour le secours de la Terre sainte. En un sens, la participation à l'expédition de Tunis fut donc la preuve de l'attachement de la noblesse au service du roi, alors même que l'idéal de la croisade ne l'animait plus. Dans les décennies 1260 et 1270, bien rares furent ceux qui, de leur propre chef, partirent outremer pour la défense de la Terre sainte. Il fallait pour cela l'encouragement donné par le roi. Au fond, à partir du moment où cet encouragement manqua, la croisade était condamnée. À tout prendre, tant Philippe III que Philippe le Bel eurent, à l'égard de la croisade, la même réticence que l'ensemble de la noblesse : il n'y eut donc pas, après Saint Louis, de divorce entre le roi et les nobles. Ils se trouvèrent implicitement d'accord.

Dans le cas de Saint Louis, l'effort de propagande tenait du chantage moral, mais il prenait appui, également, sur la notion de service du roi. Les parents du roi, ses proches et certainement aussi beaucoup de ses vassaux directs, avaient à cœur d'obéir à ses demandes, même quand ils n'y étaient pas strictement tenus. C'est ce qui passa dans les années qui précédèrent le départ pour Tunis. C'est aussi, comme on l'a vu, ce sentiment qui permit le passage d'un système d'obligations féodales strictes à un système dans lequel tous les nobles puis tous les sujets se sentaient tenus de prendre leur part à l'effort de guerre que menait, au nom de tous, le roi.

En ce qui concerne proprement la noblesse, le roi pouvait jouer sur la connivence qui l'unissait aux chevaliers. Chevalier lui-même, le roi de France, et Saint Louis comme Philippe III et Philippe le Bel, avait un crédit naturel auprès de ces derniers. Un des reproches qui fut fait à Philippe le Hardi après la chute de son favori Pierre de La Broce fut d'avoir délaissé les conseils de ses barons pour préférer ceux d'un homme de petite condition ${ }^{45}$. Une brave femme critiquait Saint Louis d'être plus un frère qu'un chevalier ${ }^{46}$. Il fallait être un homme de cabinet comme Pierre Dubois pour conseiller au roi de ne pas s'exposer ${ }^{47}$. Réciproquement, le roi se

\footnotetext{
${ }^{45}$ Chronique anonyme finissant en 1286, RHGF, t. XXI, p. 95 ; l'auteur, rendant compte des hésitations du roi à faire arrêter Pierre de la Broce et «tiex genz entour soi qui n'estoient pas preudommes ne loiaus», explique : «Mès [Philippe III] ne moustra pas tantost sa volenté ; ainz se souffri, pour ce qu'il les avoit trop creus aucunes fois. Car s'il eust dit a son conseil ne ses barons: 'Tel honte m'a fait cil', il eussent dit: 'C'est a bon droit ; vous aviez grigneur fiance en lui que vos frères'. Et por ce s'en souffri li rois ».

${ }^{46}$ «Fi ! Fi ! Deusses du estre roi de France ! Mout miex fust que un autre fust roi que tu; car tu es roy tant seulement des Freres meneurs et des Freres preecheurs et des prestres et des clers. Grant damage est que tu es roy de France, et c'est grant merveille que tu n'es bouté hors du roiaume », dit une plaignante malheureuse, Henri-François Delaborde, Vie de saint Louis par Guillaume de Saint-Pathus, confesseur de la reine Marguerite, Paris, 1890 (Collection de textes pour servir à l'étude et à l'enseignement de l'histoire), p. 118. Voyez, sur les rapports entre Saint Louis et les frères, Lester Little, «Saint Louis' Involvement with the Friars », Church History, 33 (1964), p. 125-148.

${ }^{47}$ Ch.-V. Langlois, De Recuperatione Terre sancte, Paris, Picard 1891 (Collection de textes pour servir à l'étude et à l'enseignement de l'histoire), § 118, p. 109.
} 
sentait investi d'une responsabilité éminente à l'égard non seulement du royaume, mais encore de l'armée. C'est au moins le cas pour Saint Louis, dont les états d'âme sont mieux documentés que ceux de ses successeurs. Au printemps 1250, Saint Louis eut à cœur de payer intégralement la rançon demandée par les Sarrasins pour l'armée (500 000 l. t.), plus Damiette pour sa propre délivrance ${ }^{48}$.

Dans la mise en place d'une véritable «propagande», il y a, de Saint Louis à Philippe le Bel, une forme de continuité. Le premier obtint de sa noblesse, parfois par des voies biaisées, qu'elle le suivît dans ses expéditions outremer, en recourant à l'idée de croisade, à la prédication, mais aussi aux liens qui unissaient le roi et sa noblesse. Le second mit en avant, dans le même but et avec finalement le même succès, d'autres notions, et par-dessus tout la «défense du royaume». C'est ce leitmotiv de la propagande royale qui sous-tend la généralisation du service militaire à la noblesse d'abord, à la faveur des guerres d'Aquitaine, puis à l'ensemble du royaume, avec l'apparition de la notion d' «arrière-ban ». À cette notion de « défense du royaume » était associée celle, plus discrète, de l' «honneur du roi », que ce dernier pouvait invoquer particulièrement auprès de la chevalerie. Ces motifs de la propagande royale avaient pour but de mobiliser l'opinion pour en obtenir des combattants et, peut-être surtout, de l'argent.

Sous Philippe le Bel, en effet, l'effort de propagande prit une ampleur nouvelle - plus d'ailleurs qu'il n'eut recours à des thèmes radicalement neufs. Mais il ne faut pas s'y tromper. Cet effort ne fut pas égal tout au long du règne. Au temps des victoires, dans la grande décennie 1290 , le roi et le gouvernement royal semblent s'être fort peu souciés d'obtenir l'adhésion autrement que par l'énoncé brutal de la nécessité de la «défense du royaume »- une notion étrange, du reste, comme Boniface VIII ne manqua pas de le souligner, puisque le roi, de toute évidence, avait cherché délibérément la guerre. Ce n'est qu'avec les grands barons dont il souhaitait le soutien sans être en mesure de l'exiger que Philippe le Bel se montrait caressant.

Le roi était imité en cela par les commandants de ses armées. Le 21 juillet 1296, alors qu'il dirigeait les opérations en Aquitaine, le comte d'Artois demanda « à haut et noble homme et sage son tres chier amé especial monseigneur Henri, comte de Rodés «, de venir à Langon, le 5 août suivant, avec des hommes d'armes « a tout quanques vous pourrez avoir de gens d'armes garnis de chevals et d'armes convenables «, pour aller dégager la ville de Dax assiégée, dont le capitaine avait demandé le secours du comte d'Artois ${ }^{49}$. Les formules très prudentes employées par ce dernier pour convaincre le comte de Rodez méritent d'être signalées ${ }^{50}$. Le ton dont usaient les mandements destinés aux sénéchaux ou aux baillis était nettement plus sec. Mais il changea radicalement après Courtrai. La priorité était désormais au compromis, à la négociation. Le vocabulaire employé connut une nette inflexion; le lexique de l'ordre céda devant celui de la demande, de la prière, voire de la supplique. Dans le

\footnotetext{
${ }^{48}$ Joinville, Vie de Saint Louis, éd. cit., § 343.

${ }^{49}$ BnF, coll. Doat, vol. 179, fol. 33-34 (en français) ; éd. ex ms. Colbertino, Martène, Amplissima collectio, t. I, col. 1430 (avec la date fausse de 1310).

${ }^{50}$ « Nous qui de vostre loyauté et bonté avons moult grant fiance et a bon droit, especialement a aider et garder l'honneur monseigneur le roy et son royaume, ainsi comme vous avez fait tousjours bien et loyalement a grands travaux et a grands peines, vous mandons de par monseigneur le roy et prions et requerons de par nous comme nous poons plus comme a nostre chier ami que vous si chier comme vous avez l'honneur monseigneur le roy et le vostre [...]».
} 
même temps, la notion d'arrière-ban arrivait à point nommé pour prendre le relais de la «défense du royaume», toujours usitée, de façon plus pertinente qu'auparavant, mais dont le côté spectaculaire commençait à s'atténuer pour avoir été trop mis en avant. C'est le 5 août 1302 que, pour la première fois, dans les ordres publiés par le roi, apparut l'expression d'arrière-ban. En réalité, elle n'était qu'un artifice destiné à justifier, sur le plan du droit, une convocation générale que, dans les faits, le roi était prêt à exiger, sans donner d'explications, dès le mois de juin précédent, dans un contexte alors beaucoup plus favorable pour lui. Autrement dit, l'apparition de «l'arrière-ban », complétant la notion mieux connue de «ban» (le texte dit «par ban et par arriere-ban»), donnait comme une coloration juridique à ce que, quand il en avait la possibilité quelques semaines plus tôt, le roi était disposé à imposer par l'autorité du fait accompli ; il ne se souciait guère, alors, de trouver une appellation nouvelle et légitimante à l'obligation de service enfin généralisée à tous ses sujets, point d'aboutissement d'une évolution de trente années. Devant le retournement de conjoncture, les conseillers du roi trouvèrent l'expression propre à rassurer les destinataires : il ne s'agissait pas d'une nouveauté indue, mais d'un procédé parfaitement admis, de toute ancienneté, même si le besoin ne s'en était pas fait sentir depuis longtemps. «Il n'est memoire de lonc temps que du temps de noz devanciers fust si grant besoing comme orendroit de semondre par voie d'arrere-ban, auquel toutes maniere de gent, si comme vous savez, sont tenuz a venir sanz nulle excusation $»^{51}$. «Si comme vous savez»: évidemment, personne ne savait au juste ce qu'était l'arrière-ban ; mais il ne semble pas qu'on s'en soit beaucoup préoccupé sur le moment. De toute façon, le message était clair: tous devaient courir aux armes. La noblesse semble avoir répondu volontiers à cet appel lancé par le roi, comme le fit la nation toute entière à ceux de Louis XIV (12 juin 1709) ou de la Convention (23 août 1793). L'armée réunie à l'automne 1302 fut de fait la plus nombreuse du règne. La masse qu'elle représentait et le manque de chefs de valeur pour remplacer ceux qui étaient tombés quelques semaines plus tôt suffisent à expliquer qu'elle soit restée l'arme au pied.

Par la suite, en dépit des difficultés, l'armée royale fut suffisamment nombreuse. Les inflexions données à la communication royale n'ont pas dû compter pour rien. Était-ce seulement, pour autant, le produit d'une propagande efficace? Sans doute, cet élément a-t-il joué, surtout du fait du changement spectaculaire de ton. Mais, au-delà, on peut penser que, dans la crise ouverte par le désastre militaire, une véritable conscience nationale s'est fait jour, alimentée par les efforts des services royaux, mais qui plongeait ses racines bien plus profondément - la question, naturellement, mériterait d'être étudiée de façon beaucoup plus détaillée.

À l'examen, les motivations qui ont poussé les nobles du royaume à servir dans les armées de Saint Louis, de Philippe III ou de Philippe le Bel apparaissent donc très diverses. La quête de gloire et le désir de tromper l'ennui ; le goût pour les armes et la perspective du butin, d'un établissement ou de la solde ; le service du roi et l'ombre du bailli ou du sénéchal ; ces différents sentiments, par ailleurs si difficiles à sonder, ont dû jouer, en se combinant d'une infinité de façons, chaque chevalier ou écuyer, en somme, ayant ses propres raisons de servir. La part respective de

\footnotetext{
${ }^{51}$ Arch.nat., JJ 35, fol. 30, n⿳ ${ }^{\circ} 86$; pour un relevé des occurrences de l'expression «arrièreban », Philippe Contamine, Guerre, État et société, op. cit., p. 26-27.
} 
chaque élément doit d'autre part être mise en rapport avec la chronologie et la nature de l'expédition prévue. En 1265, les motivations des chevaliers et écuyers qui suivirent Charles d'Anjou étaient à la fois les qualités propres du chef de guerre, les avantages spirituels offerts par l'Église à ces croisés d'un type nouveau, la perspective de la solde, dans l'immédiat, et celle de s'établir. À l'automne 1302, elles devaient être plutôt la fidélité et la loyauté au roi, le souci de l'honneur du royaume et quelque chose comme l'émergence d'une conscience nationale.

Au terme de cette brève réflexion, trois conclusions s'imposent.

Le roi ou ses conseillers ne se contentèrent pas d'une attitude passive. L'armée, comme la religion, accorde une grande place aux cérémonies. Les défilés, les revues, les prises d'armes, le salut au drapeau, si fortement mis en valeur au XIXe s., ont leurs équivalents au XIIIe s. La théâtralisation de l'activité militaire s'accentue à la période envisagée, au moment où l'emprise du roi sur sa noblesse devient plus forte. À partir de Saint Louis, les fêtes d'adoubement du fils aîné du roi, volontairement fastueuses, sont destinées à faire renaître le zèle en faveur de la croix (1267, 1284, 1313). L'entrée en campagne de l'ost royal est toujours précédée de la levée de l'oriflamme à Saint-Denis ${ }^{52}$. L'oriflamme, du reste, commence à revêtir la même signification qu'un véritable drapeau national. Alors qu'il raconte comment le sénéchal de Toulouse et ses hommes, assiégés dans Pampelune, attendaient l'arrivée du comte d'Artois venu à leur secours, Primat note avec un frisson empreint de patriotisme :

Mais puis que il congnurent la banière qui estoit en soie lingne, et estoit enoblie de fleurs de lis de couleurs d'or, et estoit differente des autres que le vent demenoit legièrement en deboutant par l'air, il aperçurent et sorent bien que secours leur estoit venu des parties de France ${ }^{53}$.

Ce n'est pas un hasard non plus si c'est à Mons-en-Pevèle (1304) que, pour la première fois, les combattants français arborèrent un même signe distinctif, une écharpe blanche, pour se distinguer de leurs ennemis flamands. L'effort de propagande fourni par le gouvernement royal sous Philippe le Bel et fondé sur la défense du royaume ne vint au fond que donner une autre ampleur à un phénomène déjà bien présent dans les règnes précédents, en en accentuant la dimension nationale. Il n'y a pas de solution de continuité : du reste, la présence de Philippe le Bel, à la tête de l'armée, à Mons-en-Pevèle, rappelle l'implication personnelle de Saint Louis dans le sort de l'armée capturée en Égypte ou égarée en Tunisie.

Le seconde conclusion est évidente : tous les nobles ne réagissaient pas de la même façon aux demandes du roi. Leur degré de proximité avec le roi conditionnait leur réaction. Les vassaux directs, petits et moyens, servaient, de gré ou de force, parce qu'ils le voulaient ou parce que le bailli l'exigeait. Certains d'entre eux étaient pensionnés du roi, avec les fiefs-rentes. Les hauts barons servaient eux aussi, parce que leur honneur était en jeu, et aussi leur place dans la société politique. Pour ceux qui n'étaient ni de hauts barons ni les vassaux directs du roi, ou quand l'obligation

${ }^{52}$ Philippe Contamine, L'oriflamme de Saint-Denis aux XIV et XV siècles. Étude de symbolique religieuse et royale, Nancy, 1975.

${ }_{53}^{5 H G F}$, t. XXIII, p. 95. 
de servir était absente, dans le cas des croisades par exemple, les choses étaient plus complexes, comme le montre bien le cas d'un Joinville. Celui-ci n'est pas le vassal du roi, puisqu'il est sénéchal de Champagne ; mais il est son ami. Il prend part à la croisade d'Égypte ; mais il refuse de suivre une seconde fois le roi.

Au total, donc, servir? Oui, sans doute, comme le montre le véritable sursaut national et patriotique qui suivit Courtrai. Mais il fallut quand même arrêter la guerre assez rapidement, une fois la honte de la défaite vengée à Mons-en-Pevèle. C'est la troisième conclusion : jusqu'en 1302, les rois ont été servis par une conjoncture globalement favorable. Au fond, toute une série de raisons expliquent que le roi n'eut aucune difficulté à recruter. C'est particulièrement vrai pour Philippe le Bel. Sa propagande fut efficace ; les chefs de guerre et les officiers royaux, sur place, se montrèrent attractifs, convaincants ou persuasifs. Mais, surtout, dans la décennie 1290, les campagnes furent brèves et victorieuses, et les sacrifices demandés, du coup, fort légers : si les convocations touchaient potentiellement beaucoup de gens, dans la réalité bien peu devaient partir. De plus, combien de convocations étaientelles finalement annulées ? Comme le roi payait les combattants, et probablement dès leur entrée à son service, il pouvait même être intéressant de répondre sans grogner à ses convocations. Quand survint la grande défaite de Courtrai, l'édifice construit au fil des années révéla toute sa fragilité : le contrôle du roi sur sa noblesse restait assez faible, et fondé sur l'accord, voire le compromis, surtout au fur et à mesure qu'on descendait dans la hiérarchie nobiliaire. Comme le montrera l'évolution de l'armée royale après Crécy, le consensus et l'adhésion demeuraient indispensables à la mise en place d'une véritable armée nationale.

Xavier Hélary

Université Paris IV-Sorbonne 